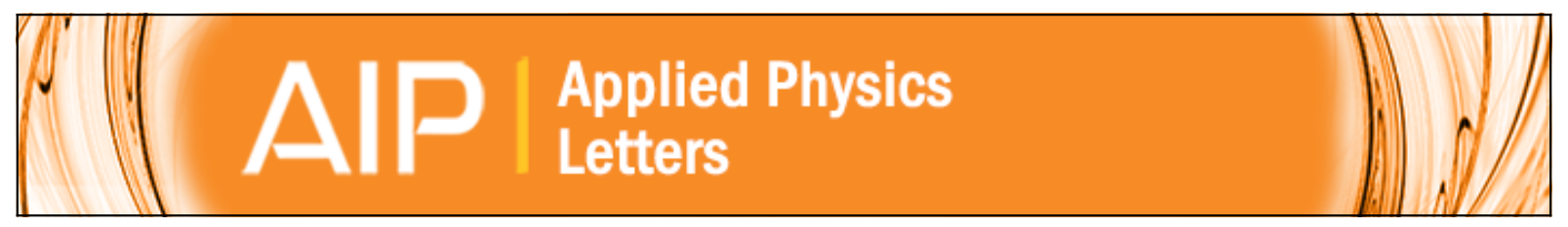

\title{
Plasmons of a two-dimensional electron gas in the presence of spin orbit interaction
} W. Xu

Citation: Applied Physics Letters 82, 724 (2003); doi: 10.1063/1.1541098

View online: http://dx.doi.org/10.1063/1.1541098

View Table of Contents: http://scitation.aip.org/content/aip/journal/apl/82/5?ver=pdfcov

Published by the AIP Publishing

\section{Articles you may be interested in}

Landau level crossing in a spin-orbit coupled two-dimensional electron gas

Appl. Phys. Lett. 106, 012106 (2015); 10.1063/1.4905492

Energy-loss rate of a fast particle in two-dimensional semiconductors with Rashba spin-orbit coupling Appl. Phys. Lett. 102, 052113 (2013); 10.1063/1.4790847

Realization of In $0.75 \mathrm{Ga} 0.25 \mathrm{As}$ two-dimensional electron gas bilayer system for spintronics devices based on

Rashba spin-orbit interaction

J. Appl. Phys. 112, 113711 (2012); 10.1063/1.4766749

Electrical manipulation of spins in the Rashba two dimensional electron gas systems

J. Appl. Phys. 105, 122402 (2009); 10.1063/1.3117232

Absorption of surface acoustic waves by a two-dimensional electron gas in the presence of spin-orbit interaction J. Appl. Phys. 94, 3229 (2003); 10.1063/1.1599631

\section{AIP $\mid$ APL Photonics \\ APL Photonics is pleased to announce Benjamin Eggleton as its Editor-in-Chief}

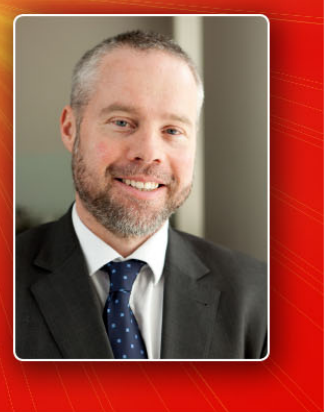




\title{
Plasmons of a two-dimensional electron gas in the presence of spin orbit interaction
}

\author{
W. $X u^{a)}$ \\ Department of Theoretical Physics, Research School of Physical Sciences and Engineering, Australian \\ National University, Canberra, ACT 0200, Australia
}

(Received 29 July 2002; accepted 3 December 2002)

\begin{abstract}
A theoretical study of the collective excitation associated with plasmon modes is presented for a two-dimensional electron gas in the presence of spin orbit $(\mathrm{SO})$ interaction induced by the Rashba effect. In such a case, the plasmon excitation can be achieved via intra- and inter-SO electronic transitions. As a result, three branches of the plasmon oscillations can be observed. It is found that inter-SO plasmons depend strongly on sample parameters and, at a long-wavelength limit, are optic-like, in contrast to intra-SO ones. The interesting features of these plasmon modes are examined. (C) 2003 American Institute of Physics. [DOI: 10.1063/1.1541098]
\end{abstract}

The recent proposals for potential applications of electronic systems with finite spin splitting at zero magnetic field to important devices such as spin-transistors, ${ }^{1}$ spin-waveguides, ${ }^{2}$ spin-filters, ${ }^{3}$ quantum computers, ${ }^{4}$ etc. have opened up a field of research known as "spintronics." It is known that in, e.g., InAs-based two-dimensional electron gas (2DEG) systems, the zero-field spin splitting can be realized from surface electric field induced by the presence of the heterostructures, known as the Rashba effect. ${ }^{5}$ The strength of the spin-orbit (SO) coupling in these systems can be controlled and altered by, e.g., applying a gate voltage, ${ }^{6}$ varying the sample growth parameters, ${ }^{7}$ etc. In order to understand these material systems more deeply and to explore their further applications to the practical devices, it is necessary and significant to examine the roles which many-body effects can play in a 2DEG with SO coupling, and it is the prime motivation of the present study.

In this letter, the case of elementary electronic excitation induced by electron-electron (e-e) interaction is considered. For a typical 2DEG in the $x y$ plane in narrow gap semiconductors, such as InGaAs/InAlAs quantum wells, the singleelectron Schrödinger equation including the lowest order of SO interaction (SOI) can be solved analytically. ${ }^{2}$ Applying the electron wave functions to the e-e interaction Hamiltonian induced by the Coulomb potential, the space Fourier transform of the matrix element for the bare e-e interaction can be obtained as

$$
V_{\alpha \beta}(\mathbf{k}, \mathbf{q})=V_{q} F_{0}(q) G_{\alpha \beta}(\mathbf{k}, \mathbf{q}) .
$$

Here, $\alpha=\left(\sigma^{\prime} \sigma\right)$ with $\sigma= \pm 1$ referring to different SOs, $\mathbf{k}$ $=\left(k_{x}, k_{y}\right)$ is the electron wave vector along the $2 \mathrm{D}$ plane, $\mathbf{q}=\left(q_{x}, q_{y}\right)$ is the change of $\mathbf{k}$ during a scattering event, $V_{q}$ $=2 \pi e^{2} / \kappa q$ with $\kappa$ being the dielectric constant, and

$$
G_{\alpha \beta}(\mathbf{k}, \mathbf{q})=\frac{1+\alpha A_{\mathbf{k q}}}{2} \delta_{\alpha, \beta}+\frac{i \alpha B_{\mathbf{k q}}}{2}\left(1-\delta_{\alpha, \beta}\right)
$$

with $A_{\mathbf{k q}}=(k+q \cos \theta) / \mathbf{k}+\mathbf{q}\left|, \quad B_{\mathbf{k q}}=q \sin \theta /\right| \mathbf{k}+\mathbf{q} \mid$, and $\theta$ being the angle between $\mathbf{k}$ and $\mathbf{q}$. Furthermore, in the present study, the case of a narrow quantum well in which only one

${ }^{a)}$ Electronic mail: wen105@rsphysse.anu.edu.au electronic subband is present is considered, thus, $F_{0}(q)$ $=\int d z_{1} \int d z_{2}\left|\psi_{0}\left(z_{1}\right)\right|^{2}\left|\psi_{0}\left(z_{2}\right)\right|^{2} \exp \left(-q\left|z_{1}-z_{2}\right|\right)$ with $\psi_{0}(z)$ being the electron wave function along the growth direction. From electron energy spectrum obtained from the solution of the Schrödinger equation, we can derive the retarded and advanced Green's functions for electrons. Applying these Green's functions and $V_{\alpha \beta}(\mathbf{k}, \mathbf{q})$ to the diagrammatic techniques to derive effective $\mathrm{e}-\mathrm{e}$ interaction under the randomphase approximation, the element of the dielectric function matrix is obtained as

$$
\epsilon_{\alpha \beta}(\Omega, q)=\delta_{\alpha, \beta}-\sum_{\mathbf{k}} V_{\alpha \beta}(\mathbf{k}, \mathbf{q}) \Pi_{\beta}(\Omega ; \mathbf{k}, \mathbf{q}),
$$

where

$$
\Pi_{\sigma^{\prime} \sigma}(\Omega ; \mathbf{k}, \mathbf{q})=\frac{f\left[E_{\sigma^{\prime}}(\mathbf{k}+\mathbf{q})\right]-f\left[E_{\sigma}(\mathbf{k})\right]}{\hbar \Omega+E_{\sigma^{\prime}}(\mathbf{k}+\mathbf{q})-E_{\sigma}(\mathbf{k})+i \delta}
$$

is the pair bubble in the absence of e-e coupling and $f(E)$ is the Fermi-Dirac function. Furthermore

$$
E_{\sigma}(\mathbf{k})=E_{\sigma}(k)=\hbar^{2} k^{2} / 2 m^{*}+\sigma \alpha_{R} k
$$

is the energy spectrum of a 2DEG in the presence of SOI, with $m^{*}$ being the electron effective mass and $\alpha_{R}$ being the Rashba parameter which measures the strength of the SOI. Because $\Sigma_{\mathbf{k}} B_{\mathbf{k q}} \Pi_{\sigma^{\prime} \sigma}(\Omega ; \mathbf{k}, \mathbf{q})=0$, the dielectric function matrix of a 2DEG with Rashba spin splitting is obtained, setting $1=(++), 2=(+-), 3=(-+)$ and $4=(--)$, as

$$
[\epsilon(\Omega, q)]=\left(\begin{array}{cccc}
1+a_{1} & 0 & 0 & a_{4} \\
0 & 1+a_{2} & a_{3} & 0 \\
0 & a_{2} & 1+a_{3} & 0 \\
a_{1} & 0 & 0 & 1+a_{4}
\end{array}\right) .
$$

In Eq. (3), $a_{j}=-\left[V_{q} F_{0}(q) / 2\right] \Sigma_{\mathbf{k}}\left(1 \pm A_{\mathbf{k q}}\right) \Pi_{j}(\Omega ; \mathbf{k}, \mathbf{q})$ where upper (lower) case refers to $j=1$ or 4 for intra-SO transitions ( $j=2$ or 3 for inter-SO transitions). The determinant of the dielectric function matrix is

$$
|\epsilon(\Omega, q)|=\left(1+a_{1}+a_{4}\right)\left(1+a_{2}+a_{3}\right) .
$$

The modes of elementary electronic excitations are determined by $\operatorname{Re}|\epsilon(\Omega, q)| \rightarrow 0$. In the presence of SOI, the col- 


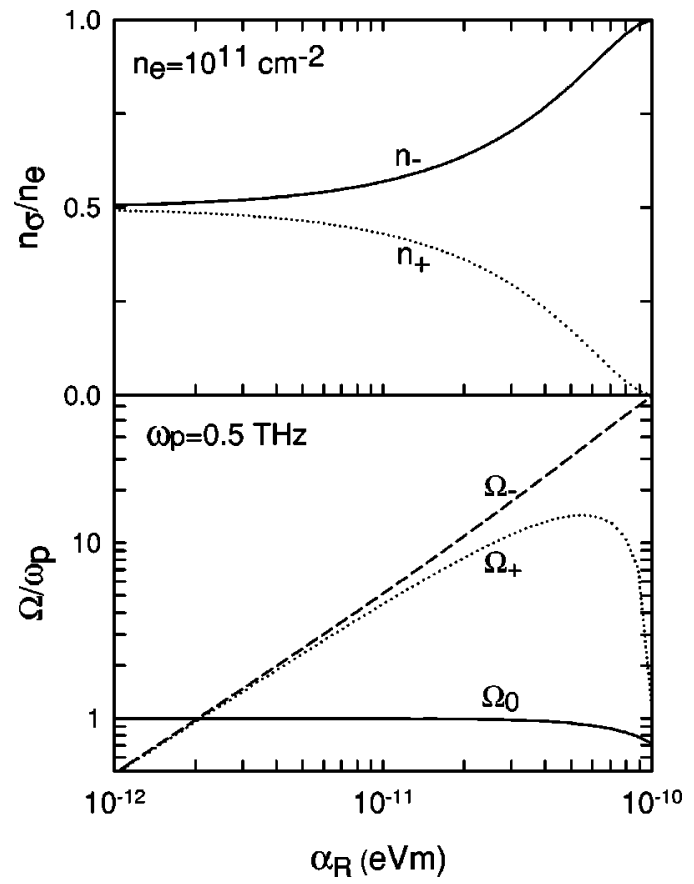

FIG. 1. Electron density in different SOs $\left(n_{\sigma}\right)$ and plasmon frequency $\left(\Omega_{0}\right.$ and $\Omega_{+}$induced, respectively, by intra- and inter-SO excitation) vs the Rashba parameter $\left(\alpha_{R}\right)$ at a fixed total electron density $n_{e}$ and a fixed $\omega_{p}$ $=\left(2 \pi e^{2} n_{e} q / \kappa m^{*}\right)^{1 / 2}$.

lective excitation can be achieved via electron transitions in different spin channels. At long wavelength (i.e., $q \ll 1$ ) and low temperature (i.e., $T \rightarrow 0$ ) limit, the plasmon frequencies induced by intra- and inter-SO excitation are given, respectively, by

$$
\Omega_{0}=\omega_{p}\left(1-\frac{\omega_{-}-\omega_{+}}{\omega_{0} / 2}\right)^{1 / 2},
$$

and by solving

$$
\ln \left(\frac{\Omega+\omega_{-}}{\Omega-\omega_{-}} \cdot \frac{\Omega-\omega_{+}}{\Omega+\omega_{+}}\right)=\frac{\omega_{0} \Omega}{\omega_{p}^{2}} .
$$

Here, $\omega_{p}=\left(2 \pi e^{2} n_{e} q / \kappa m^{*}\right)^{1 / 2}$ is the plasmon frequency of a 2DEG in the absence of the SOI, $\omega_{0}=16 \pi n_{e} \hbar / m^{*}, \omega_{ \pm}$ $=4 \alpha_{R} \sqrt{\pi n_{ \pm}} / \hbar$ and $n_{e}=n_{+}+n_{-}$is the total electron density of the 2DEG. Moreover, the electron density in different spin channels

$$
n_{ \pm}=\left(n_{e} / 2\right) \mp\left(k_{\alpha} / 2 \pi\right) \sqrt{2 \pi n_{e}-k_{\alpha}^{2}}
$$

is obtained from the condition of electron number conservation for case of $n_{e}>k_{\alpha}^{2} / \pi$ with $k_{\alpha}=m^{*} \alpha_{R} / \hbar^{2}$. When $n_{e}$ $\leqslant k_{\alpha}^{2} / \pi$, only spin-down states are occupied by electrons and, therefore, the system is spin polarized (i.e., $n_{+}=0$ and $\left.n_{-}=n_{e}\right)$. It can be seen from Eq. (5b) that at a longwavelength limit, two branches of the inter-SO plasmon can be observed when $\Omega_{ \pm} \rightarrow \omega_{ \pm}$and $\Omega_{ \pm}$depends generally on $q$ via $\omega_{p}$.

In Fig. 1, the electron density in different SOs and plasmon frequency induced by intra- and inter-SO excitation are shown as a function of the Rashba parameter at a fixed $n_{e}$ and a fixed $\omega_{p}$ for an InGaAs-based 2DEG. The nature of the energy spectrum for a 2DEG with SOI [see Eq. (2c)] implies that although the direction of the spin can change when an electron moves in $\mathbf{k}$ space, the density of states (DOS) for

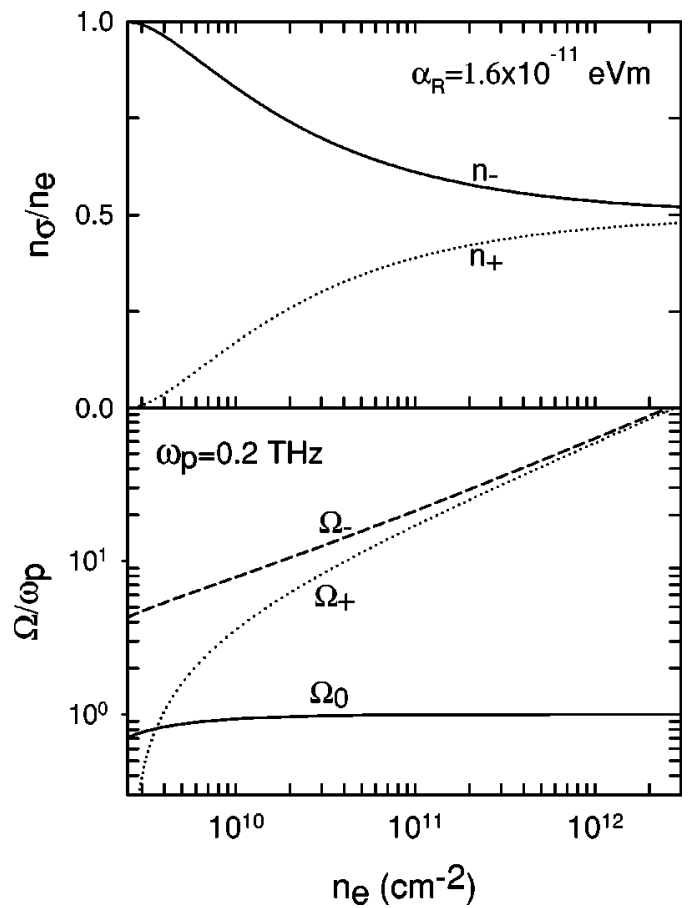

FIG. 2. Electron distribution and plasmon frequency as a function of total electron density for fixed $\alpha_{R}$ and $\omega_{p}$.

spin-up channel is shifted to the higher energy regime in comparison to that for spin-down one. Thus, the electron DOS and, consequently, the electron distribution in different SOs can be different. With increasing $\alpha_{R}$, more and more electrons are in the spin-down channel because it has a lower energy. A relatively big difference between $n_{-}$and $n_{+}$has been measured experimentally. ${ }^{7}$ We see from Fig. 1 that: (1) $\Omega_{0}$ induced by intra-SO excitation differs from $\omega_{p}$ and decreases slowly with increasing $\alpha_{R}$ [also see Eq. (5a)]; (2) $\Omega_{-}$ induced by inter-SO excitation increases almost linearly with $\alpha_{R}$; (3) $\Omega_{+}$induced by inter-SO excitation as well first increases then decreases with increasing $\alpha_{R}$; (4) $\Omega_{ \pm}$can be much larger than $\Omega_{0}$ and $\Omega_{-}$is always larger than $\Omega_{+}$, which indicates that high-frequency plasmon excitation can be achieved in a spin-split 2DEG via inter-SO electronic transitions; (5) the larger differences between $n_{-}$and $n_{+}$, $\Omega_{0}$ and $\omega_{p}$, and between $\Omega_{-}$and $\Omega_{+}$can be observed at a larger value of $\alpha_{R}$; and (6) $\Omega_{ \pm}$depends much more strongly on $\alpha_{R}$ than $\Omega_{0}$ does.

In Fig. 2, the electron distribution in different SOs and plasmon frequency due to different excitation modes are plotted as a function of total electron density for fixed $\alpha_{R}$ and $\omega_{p}$. The more pronounced differences between $n_{-}$and $n_{+}, \Omega_{-}$and $\Omega_{+}$, and between $\Omega_{0}$ and $\omega_{p}$ can be seen at relatively lower electron densities. $\Omega_{-} / \omega_{p}$ increases almost linearly with $n_{e}$, whereas $\Omega_{+} / \omega_{p}$ increases more rapidly with $n_{e}$. Again, $\Omega_{ \pm}$depends much more strongly on $n_{e}$ than $\Omega_{0}$ does and $\Omega_{ \pm}$can be much larger than $\Omega_{0}$. Since plasmon excitation from an electron gas is achieved by electronic transition around Fermi level $\left(E_{F}\right)$, changing sample parameters such as $\alpha_{R}$ and $n_{e}$ implies that $E_{F}$ is varied and, so, $\Omega_{0}$ and $\Omega_{ \pm}$depend on $\alpha_{R}$ and $n_{e}$. Furthermore, a nonparabolic subband structure of the 2DEG with SOI [see Eq. (2c)] leads to an $\Omega_{0}$ different from that obtained from a parabolic one [see Eq. (5a)]. 


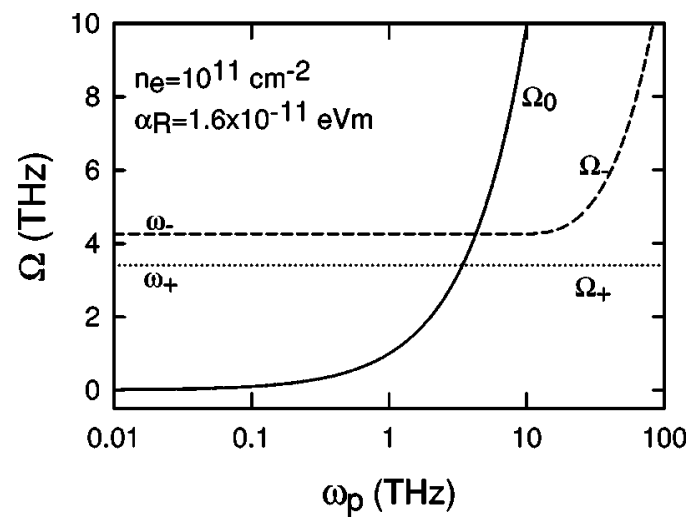

FIG. 3. Dependence of the plasmon frequency on $\omega_{p}=\left(2 \pi e^{2} n_{e} q / \kappa m^{*}\right)^{1 / 2}$ for fixed $\alpha_{R}$ and $n_{e}$. Here $\omega_{ \pm}=4 \alpha_{R} \sqrt{\pi n_{ \pm}} / \hbar$ and note different scales for $\Omega$ and $\omega_{p}$.

The dependence of plasmon frequency due to intra- or inter-SO excitation on $\omega_{p}$ or $q$ is shown in Fig. 3 for fixed $\alpha_{R}$ and $n_{e}$, where $q$ is the change of electron wave vector (or momentum) during a scattering event. At a longwavelength limit, $\Omega_{0} \sim \omega_{p} \sim q^{1 / 2}$ [see Eq. (5a)] similar to the case in the absence of the SOI. Although $\Omega_{ \pm}$should, in principle, depend on $q$ via $\omega_{p}$ [see Eq. (5b)], the numerical results shown here suggest that at a long-wavelength limit, over a wide regime of $\omega_{p}$ or $q, \Omega_{ \pm} \rightarrow \omega_{ \pm}=4 \alpha_{R} \sqrt{\pi n_{ \pm}} / \hbar$ depends very little on $q$ or $\omega_{p}$, especially for $\Omega_{+}$. The most important conclusion drawn from Fig. 3 is that at longwavelength limit, inter-SO plasmons are optic-like, in sharp contrast to intra-SO plasmon and to those observed in a conventional 2DEG. It should be noted that $\alpha_{R}$ and $n_{e}$ used in Fig. 3 are typical sample parameters realized from InGaAs/ InAlAs quantum wells. ${ }^{6,7}$ For these parameters, $\Omega_{ \pm} \sim \mathrm{THz}$ and $\Omega_{-}-\Omega_{+} \sim 1 \mathrm{THz}$ (or $4 \mathrm{meV}$ or $300 \mu \mathrm{m}$ ) can be achieved, which suggests that these plasmon modes are measurable by using recently developed long-wavelength laser and optoelectronic technologies.

In this study, it has been found that in a 2DEG with SOI due to the Rashba effect, the plasmon excitation can be achieved via intra- and inter-SO electronic transitions so that three plasmon modes can be generated. The inter-SO plasmons $\left(\Omega_{-}\right.$and $\left.\Omega_{+}\right)$depend strongly on the strength of the SOI and total electron density of the system, whereas the intra-SO one $\left(\Omega_{0}\right)$ depends much more weakly on these sample parameters. The plasmon frequency (especially $\Omega_{ \pm}$) of a 2DEG in the presence of SOI can differ significantly from $\omega_{p}$ obtained from a conventional 2DEG. In InAs-based 2DEGs, $\Omega_{ \pm}$and $\Omega_{-}-\Omega_{+}$can reach up to THz. This suggests that these systems can be used as semiconductor $\mathrm{THz}$ generators and detectors. At long-wavelength limit, in sharp contrast to $\Omega_{0} \sim \omega_{p} \sim q^{1 / 2}, \Omega_{ \pm}$depends very little on $q$ and therefore is optic-like. Experimentally, it is not so easy to measure $\omega_{p}$ for a 2DEG because $\omega_{p} \sim q^{1 / 2}$, where techniques such as grating couplers have to be used. ${ }^{8}$ However, opticlike plasmons excited via inter-SO transitions in a $2 \mathrm{DEG}$ should be more easily observed through, e.g., optical absorption, ${ }^{9}$ Raman spectrum, ${ }^{10}$ ultrafast pump-and-probe experiments, ${ }^{8}$ etc. I therefore hope that the theoretical predictions in this letter can be verified experimentally.

The author is a Research Fellow of the Australian Research Council. Discussions with P. Vasilopoulos (Concordia, Canada) and M. P. Das (ANU, Australia) are gratefully acknowledged.

${ }^{1}$ B. Datta and S. Das, Appl. Phys. Lett. 56, 665 (1990).

${ }^{2}$ X. F. Wang, P. Vasilopoulos, and F. M. Peeters, Phys. Rev. B 65, 165217 (2002).

${ }^{3}$ T. Koga, J. Nitta, H. Takayanagi, and S. Das, Phys. Rev. Lett. 88, 126601 (2002).

${ }^{4}$ See, e.g., Y. Ohno, D. K. Young, B. Beschoten, F. Matsukura, H. Ohno, and D. D. Awschalom, Nature (London) 402, 790 (1999).

${ }^{5}$ E. I. Rashba, Sov. Phys. Solid State 2, 1109 (1960).

${ }^{6}$ J. Nitta, T. Akazaki, H. Takayanagi, and T. Enoki, Phys. Rev. Lett. 78, 1335 (1997).

${ }^{7}$ J. Luo, H. Munekata, F. F. Fang, and P. J. Stiles, Phys. Rev. B 41, 7685 (1990).

${ }^{8}$ See, e.g., M. Voßebürger, H. G. Roskos, F. Wolter, C. Waschke, and H. Kurz, J. Opt. Soc. Am. B 13, 1045 (1996).

${ }^{9}$ See, e.g., S. J. Allen, Jr., D. C. Tsui, and R. A. Logan, Phys. Rev. Lett. 38, 980 (1977).

${ }^{10}$ See, e.g., D. Olego, A. Pinczuk, A. C. Gossard, and W. Wiegmann, Phys. Rev. B 25, 7867 (1982). 